\title{
L'émergence de la violence dans la manifestation de rue. Eléments pour une analyse étiologique
}

\section{Olivier Fillieule}

\section{(2) OpenEdition \\ Journals}

Édition électronique

URL : http://journals.openedition.org/conflits/212

DOI : $10.4000 /$ conflits.212

ISSN : $1777-5345$

Éditeur :

CCLS - Centre d'études sur les conflits lilberté et sécurité, L'Harmattan

Édition imprimée

Date de publication : 15 mai 1993

ISSN : 1157-996X

\section{Référence électronique}

Olivier Fillieule, «L'émergence de la violence dans la manifestation de rue. Eléments pour une analyse étiologique », Cultures \& Conflits [En ligne], 09-10 | printemps-été 1993, mis en ligne le 04 mars 2005 consulté le 30 mars 2021. URL : http://journals.openedition.org/conflits/212 ; DOI : https://doi.org/ $10.4000 /$ conflits. 212

Ce document a été généré automatiquement le 30 mars 2021 


\title{
L'émergence de la violence dans la manifestation de rue. Eléments pour une analyse étiologique
}

\author{
Olivier Fillieule
}

\section{UN BESOIN DE THEORIE}

2 A la question de savoir pourquoi, quand et comment les violences surviennent dans des situations données, les modèles théoriques classiques ${ }^{1}$ ne sont pas parvenu à apporter une réponse réellement satisfaisante, à la fois en termes de propositions théoriques et de validations empiriques. A cela, nous voyons plusieurs raisons. D'abord, ces modèles déterminent un grand nombre de facteurs qui ont tous quelque chose à voir avec l'explication des violences mais s'en tiennent à une accumulation de variables indépendantes reproduisant la complexité du réel dans l'ordre théorique. Aucun facteur déterminant n'apparait donc clairement. Il ne fait pas de doute que les inégalités, les demandes insatisfaites et les clivages sociaux se traduisent constamment par un certain degré de conflit. Ce qui demeure mystérieux, et ce qui doit faire l'objet de l'investigation, c'est la nature profonde du lien existant entre une série de causes imbriquées et l'événement violent. Pourquoi, tout simplement, des conditions similaires ne produisent-elles pas toujours les mêmes effets? Le niveau d'étude auquel se placent la plupart des chercheurs est tellement large que l'agrégation des données débouche sur de fortes incertitudes méthodologiques ${ }^{2}$. Les hypothèses posées par les différents modèles induisent des comparaisons internationales sur le long terme, une mise en rapport de l'occurrence des violences avec l'état de la société (système politique, etc.). La recherche de la cause finit par se diluer dans le flou des données recueillies. Cette confusion dans l'ordre théorique est renforcée par une méthode presque toujours inductive dans l'exploitation des sources. La plupart du temps les données sont adaptées au modèle et c'est une des raisons pour laquelle des résultats 
différents sont produits à partir de mêmes données ${ }^{3}$. Enfin, on note une grande confusion taxinomique avec des définitions de la violence collective s'étendant de la grève aux révolutions: selon les auteurs, on travaillera sur les "luttes civiles"4, la "violence collective" ${ }^{15}$, les "révolutions"6 ou tout simplement "la violence"7. Par ailleurs, même lorsque les niveaux d'analyse sont identiques, on trouve quelquefois sous les mêmes définitions des événements qui ne sont ni collectifs (les assassinats politiques, par exemple) ni violents (les élections, certaines manifestations pacifiques). Si l'on tient compte de ces critiques, il apparaît nécessaire de resserrer à la fois l'objet d'étude, à savoir le matériel utilisé, le niveau d'analyse et la définition. C'est ce que nous nous proposons de faire ici en travaillant sur les dossiers établis par les CRS à l'occasion de manifestations survenues entre 1982 et $1990^{\circ}$. Il s'agira donc moins d'étudier les types d'événements pouvant donner lieu à des violences que d'analyser l'émergence de la violence dans une catégorie donnée d'événements, les manifestations.

Une perspective rationnelle renouvelée Les rapports rédigés par les CRS sont des récits de manifestation vus du côté policier. Cela veut dire que l'on ne dispose pas d'analyses en amont de la manifestation (données sur les organisations impliquées, sur leur histoire, sur l'inscription de l'événement dans une stratégie d'ensemble). Par ailleurs, les renseignements sur les effets de la manifestation en termes de gains ou de pertes sont limités aux effets immédiats. La seule unité d'analyse possible dans ces conditions est l'événement violent. Il y a donc bien un changement d'optique par rapport aux travaux axés sur la recherche d'explications à trouver en dehors même du "moment/manifestation"'. C'est au nom de cette orientation théorique centrée sur les processus plutôt que sur les déterminations que nous choisissons de limiter l'analyse des violences au moment même de la manifestation. Cela implique clairement que l'on se place dans une perspective rationnelle, théoriquement assez proche de celle de la mobilisation des ressources. En effet, travailler sur le moment/manifestation implique la volonté délibérée de laisser de côté les déterminants lointains de l'action violente. Qu'il s'agisse de la frustration, de l'effet des changements économiques ou de la modernisation, ces variables sont toutes mises en oeuvre par le modèle psychologique. L'analyse des dossiers CRS permet d'échapper à ce travers en "désagrégeant" le niveau d'analyse par la diminution du nombre de facteurs à prendre en compte dans l'explication. La focalisation de l'attention sur l'événement violent permet encore de prendre en considération l'échange de coups $^{10}$ et l'adaptation de chacun des protagonistes dans le temps même de la manifestation. Autrement dit, tout n'est pas joué à l'avance et la violence est bien souvent le produit d'un processus itératif d'adaptation tactique ${ }^{11}$. Ce qui nous conduit à privilégier l'étude qualitative d'un corpus de manifestations singulières à partir de l'observation des interactions entre les différents agents et l'environnement immédiat. Nous considérons d'autre part que la violence constitue un recours normal de l'action collective, même s'il s'agit d'une attitude extrême. Dans le cadre des manifestations, l'usage de la violence s'inscrit dans une chaîne d'actions ramifiées qui utilise tout l'éventail des possibilités légales et extralégales ${ }^{12}$. Pourtant, nous nous démarquons sensiblement de la perspective de la mobilisation des ressources par l'importance que nous accordons aux contextes, par le refus d'une distinction de nature entre le jeu des acteurs mobilisés et celui des agences de contrôle, par la prise en compte de l'hétérogénéité des groupes en présence et par la reconnaissance des "faits de hasard" dans le déclenchement des violences. La prise en compte des aspects stratégiques du comportement des acteurs ne signifie pas que ceuxci agissent dans un vacuum social. Pourtant, les théories de la mobilisation des 
ressources, surtout dans leur version "économiste", ne tiennent pas toujours compte du poids des contextes dans l'analyse des mobilisations. Or, nous sommes convaincu que ni la violence politique ni la manifestation pacifique ne peuvent être expliquées sans référence aux conditions sociales du moment et à la structure des opportunités disponibles pour chacun des protagonistes. Pour expliquer pourquoi telle ou telle stratégie est adoptée, il est indispensable de dire pourquoi toutes les autres n'ont pas été choisies. Par ailleurs, si les stratégies des mouvements, leurs chances de succès et le risque d'apparition de la violence sont fortement contraints par leur environnement, les mouvements modifient en retour cet environnement, le transformant quelquefois sensiblement. Là encore, plutôt que de raisonner en termes de relations monocausales et univoques, il semble plus pertinent de parler de processus interactif ${ }^{13}$. Nous pensons également qu'il n'est pas illégitime de mettre sur le même plan les stratégies des mouvements et celles de l'appareil d'Etat. Contrairement à ce que suggère par exemple Oberschall ${ }^{14}$, l'activité tactique des différents protagonistes est de même nature et doit s'analyser dans les mêmes termes. Les théoriciens de la mobilisation des ressources ont cru voir une différence de nature là où il n'y a qu'une différence de niveau de ressources. D'ailleurs, admettre une identité de nature entre les adversaires ne revient pas à nier les différences de répertoire d'action et de stock de ressources ${ }^{15}$. Il nous semble également nécessaire de tenir compte de l'hétérogénéité des groupes en présence en évitant de parler des "forces de l'ordre" ou des "manifestants" comme d'entités unifiées, d'autant que cette hétérogénéité peut se trouver à l'origine de la violence. Du côté des manifestants, les divergences sur les buts à atteindre, les différences de moyens mis en œuvre et quelquefois de cultures politiques font que les manifestants marchent rarement comme un seul homme. A la "dispersion" des éléments mobilisés correspond enfin, dans une certaine mesure, la diversité des forces de l'ordre engagées sur le terrain ${ }^{16}$, diversité dont nous verrons qu'elle peut avoir des effets non négligeables dans le déclenchement des violences. L'analyse des dossiers CRS nous suggère enfin de redonner sa place à l'événement fortuit dans l'explication. Il faut admettre selon nous l'impossibilité pratique de tout faire entrer dans un modèle et nous essaierons de montrer comment, sous l'effet de l'interaction entre les différents agents, se déclenchent des événements imprévisibles dont personne n'est maitre et qui peuvent être à l'origine des violences.

4 La question de la définition Etant donné que la violence s'identifie par rapport aux normes qu'elle enfreint, on peut s'attendre à ce que sa définition se heurte à des enjeux socialement construits. Toute définition de l'action violente dépend des normes en vigueur qui donnent leur contenu à la notion. Il en résulte logiquement une forte subjectivité et relativité des définitions en fonction des groupes en charge de la qualification ${ }^{17}$. Aussi est-il nécessaire de rejeter l'illusion selon laquelle il serait possible de donner un sens unitaire à une mobilisation, pour au contraire considérer les significations évaluatives défendues par chacun comme un élément parmi d'autres de

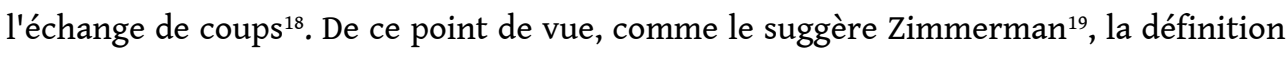
de Nieburg est sans doute la plus pertinente. Selon ce dernier, la violence politique regroupe "les actes se traduisant par des destructions, des atteintes physiques, dont le but, le choix des cibles ou des victimes, la mise en oeuvre et/ou l'effet, ont une signification politique, c'est-à-dire, tendent à modifier la conduite des protagonistes dans une situation de négociation qui à des conséquences pour le système social" ${ }^{20}$. Dans notre analyse des manifestations, nous restons assez proche de cette définition et nous considérons comme violence politique toute action protestataire et/ou policière 
dans laquelle la réunion de plusieurs personnes à des fins politiques (ou, si l'on préfère, revendicatives) a pour effet de provoquer des blessures corporelles et/ou des dégâts matériels dans le domaine public ou privé et tend à modifier la position des protagonistes dans une situation d'échange de coups. Cette définition "par l'effet" présente plusieurs avantages: elle permet d'insister sur le rôle stratégique de l'usage de la violence comme moyen de négociation; elle décrit la violence comme un processus interactif qui se joue entre plusieurs groupes d'acteurs; elle prend en compte aussi bien la violence des groupes protestataires que celle de l'Etat et indique qu'il n'est pas possible d'étudier la violence dans les mobilisations sans tenir compte des interactions entre forces de l'ordre et manifestants; enfin, elle inclut à la fois les violences préméditées et celles non voulues à l'avance, produites par le jeu imprévisible de l'interaction entre agents. A partir de ces orientations méthodologiques, on peut circonscrire l'analyse étiologique aux facteurs lisibles dans l'interaction entre les groupes antagonistes. On s'en tiendra ici à un aperçu, avec d'un côté les déterminants macrosociologiques, c'est-à-dire les facteurs propres aux groupes et, de l'autre, les déterminants microsociologiques regroupant les facteurs morphologiques qui caractérisent le "moment manifestant".

\section{FACTEURS LIES AUX GROUPES}

Les groupes de manifestants De 1982 à 1990, le groupe faisant le plus souvent l'objet d'un rapport pour emploi de la force est d'abord celui des agriculteurs (39\%). Viennent ensuite les salariés d'entreprises en difficulté, mobilisés soit contre un plan de licenciement, soit contre la cessation des activités de leur entreprise (19\%). Dans cette catégorie, on trouve surtout les salariés des chantiers navals (NORMED, Dubigeon, etc.), des aciéries de Lorraine et des mines (Potasse, charbon). Si l'on ajoute les manifestations violentes d'artisans et petits commerçants, il apparaît clairement que les manifestations violentes sont le fait des secteurs économiques les plus sinistrés de l'économie française. Quant aux étudiants et lycéens, ils représentent $14 \%$ de notre corpus, devant les manifestations organisées par les groupes nationalistes corse et basque (10\%). Il est frappant de constater que les manifestations à caractère politique, qu'il s'agisse de revendications de politique intérieure ou internationale, sont quasiment absentes de notre corpus. De même, les manifestations d'étrangers (interdites, en droit) sont peu nombreuses. La violence semble donc caractériser plutôt les manifestations dont les revendications sont de caractère économique. Toutefois, avant de pouvoir parler d'une spécificité de telle ou telle catégorie, il faut encore savoir si les groupes les plus violents sont aussi les plus manifestants, afin de maîtriser les effets de taille. Or, la comparaison des télégrammes d'emploi et des rapports techniques ${ }^{21}$ fait apparaître que les groupes désignés précédemment sont effectivement plus violents que la moyenne. Par exemple, si 39\% des manifestations violentes dans les rapports techniques sont dues aux agriculteurs, cela n'est vrai que pour $6 \%$ des télégrammes d'emploi en 1989 et 19\% en 1990. Il en va de même, bien que moins nettement, pour les salariés d'entreprises en difficulté et les artisans et petits commerçants. La même remarque vaut encore pour les étudiants et les groupes autonomistes. Autre confirmation, cette fois a contrario : les manifestations émanant du secteur public ou des entreprises publiques sont très nombreuses dans les 
télégrammes d'emploi alors même qu'elles ne concernent aucune des manifestations violentes recensées dans les rapports techniques. La violence tiendrait donc moins au caractère économique des revendications qu'à la position des groupes dans leur environnement. Au total, on peut tirer les conclusions suivantes, en termes de fréquence et non d'intensité des violences : les agriculteurs, les salariés d'entreprise en difficulté, les artisans et petits commerçants, les étudiants et les groupes autonomistes ont des pratiques qui débouchent plus souvent que la moyenne sur des violences; les employés du secteur public (salariés de l'Etat ou d'entreprises publiques) ont une pratique manifestante très fréquente mais beaucoup plus pacifique que la moyenne ; les manifestations politiques (politique intérieure ou politique internationale) sont la plupart du temps non violentes. Il en va de même pour les manifestations d'étrangers ${ }^{22}$. A partir de ces premiers résultats, peut-on déterminer une ou plusieurs explications à ce recours variable à la violence selon les groupes sociaux? Nous examinerons, parmi d'autres, les deux hypothèses suivantes : la nature des objectifs et l'existence de répertoires d'action spécifiques.

6 Nature des objectifs : les objectifs poursuivis par un groupe donné peuvent-ils être déterminants de l'apparition d'incidents violents ? Nous avons vu déjà que le caractère économique, revendication professionnelle ou de statut, ou extra-économique des revendications -commémoration, soutien à une cause étrangère, réclamation d'un droit nouveau, affirmations idéologiques diverses - permettait d'établir des corrélations avec la survenance des violences, mais sans qu'il soit possible de dire si le facteur déterminant était la nature de la revendication ou la position du groupe dans son environnement. J. A. Franck ${ }^{23}$ opère une distinction entre les objectifs "ordinaires", c'est-à-dire "acceptables" et réalisables et les objectifs "excentriques", "inacceptables", c'est-à-dire remettant globalement en cause les valeurs fondamentales de la société ou le pouvoir existant. Par rapport à la distinction économique/extra économique, sa typologie a le mérite de tenir compte aussi du statut du groupe dans son environnement. Or, dans notre corpus, $76 \%$ des incidents s'accompagnent de revendications dont les protagonistes ne sont pas sûr du tout qu'elles seront entendues, soit qu'elles soient inacceptables pour le pouvoir, dans le cas des autonomistes par exemple, soit qu'elles soient "irréalistes", c'est-à-dire impossibles à satisfaire. C'est le cas notamment des manifestations d'employés licenciés, des travailleurs des chantiers navals de la Seyne et de la Ciotat, des mineurs du nord. Il ne fait donc pas de doute, à considérer les dossiers CRS, que la nature des revendications est un facteur déterminant dans l'apparition des violences collectives. Reste à interpréter ce lien entre certains types de revendications et l'occurrence des violences. On serait tenté de dire que l'absence d'issue possible, c'est-à-dire de solution négociée avec concessions mutuelles, l'impression que l'on n'a plus rien à perdre, un sentiment exacerbé d'injustice, contribueraient largement au déclenchement des violences. Ce point suggère qu'il est peut-être nécessaire d'isoler, dans certains cas, un type de violence ne ressortissant pas de l'utilisation stratégique. La violence ne s'inscrirait plus alors dans le cadre d'une riposte graduée et viendrait clore, d'une certaine façon, la lutte ${ }^{24}$. En même temps qu'une fonction de "réassurance, de réarmement moral" de groupes touchés dans leur existence même, dans leur légitimité à être, la violence remplirait aussi une fonction idéologique. Selon Wilson ${ }^{25}$, une idéologie comprend un diagnosis (une détermination des causes du mécontentement et une imputation de responsabilité à un acteur donné), un prognosis (une indication de ce qui doit être fait) et un rationale (détermination de qui doit agir, quels arguments utiliser pour convaincre, etc...). 
Plusieurs auteurs ont montré combien il était nécessaire pour un mouvement d'agir sur ces trois aspects de l'idéologie afin de redéfinir les situations et de marquer une rupture avec les discours justifiant le statu quo ${ }^{26}$. Or, l'usage de la violence a pour effet de contribuer à la définition d'une situation comme injuste, puisque elle constitue "un langage qui dramatise la situation et unifie les perceptions. En rendant réel l'affrontement, elle conteste les représentations dominantes en termes de fatalité" ${ }^{27}$. De fait, l'usage de la violence participe de la construction et de la gestion du sens par les groupes mobilisés. S'il est vrai que les plus menacés dans leur existence sont aussi les plus violents, cela ne veut pas dire pour autant que leur violence est erratique et sans utilité tactique prévue à l'avance. Lors d'une manifestation d'agriculteurs par exemple, la violence permet aussi au groupe de rompre avec les représentations dominantes qui voudraient que la disparition de l'agriculture soit obligatoirement le produit d'une logique économique non maîtrisable; d'abord en résistant à ladite logique de façon "manifeste", mais aussi en désignant des responsables par le choix des cibles de la violence. Enfin, puisque les groupes sociaux ont un recours différent à la violence collective, il est légitime de se demander si ces différences ne s'expliquent pas, en partie au moins, par un usage plus ou moins habituel de la manifestation ou par des répertoires d'action spécifiques.

Répertoires d'action: on peut supposer que moins le groupe de manifestants a un recours usuel à la manifestation et plus souvent celle-ci peut "dégénérer". Les groupes sociaux auraient comme une "mémoire manifestante" : les catégories qui manifestent de longue date, ouvriers, fonctionnaires le feraient généralement dans le calme, alors que les groupes qui se mobilisent depuis peu de temps ne sauraient pas encore manifester dans les règles, d'où la probabilité d'incidents. C'est que la pratique de la manifestation s'assortit d'un certain nombre de techniques et de règles implicites élaborées au cours du temps dont tous les agents sociaux n'ont pas immédiatement la maitrise. Que l'on songe notamment à l'organisation d'un service d'ordre capable d'éviter les provocations ou les accidents, au travail de négociation permanent qu'il faut faire sur le terrain avec le représentant des forces de l'ordre pour gérer le bon déroulement de la manifestation (négociation de l'itinéraire, modalités de réception des délégations, etc...) ${ }^{28}$. On peut également faire l'hypothèse, avérée par de nombreux travaux historiques, qu'il existe des traditions de recours à la violence politique chez certains groupes sociaux. Plusieurs travaux portant sur les révolutions en Europe au $\mathrm{XVIII}^{\circ}$ et $\mathrm{XIX}^{\circ}$ siècles ont souligné la fréquence avec laquelle les ouvriers et les boutiquiers parisiens descendirent dans la rue pendant la révolution de 1789 et après, jusqu'en 1795 , en 1830,1848 et $1871^{29}$. Les traditions de violence sont encore plus aisément décelables dans les milieux ruraux. Par exemple, les émeutes de la faim furent un trait caractéristique des campagnes anglaises au XVIII ${ }^{\circ}$ siècle et il y en eu près de 275 entre 1735 et 1800, en étroite relation avec les mauvaises récoltes ou la chute du prix du grain ${ }^{30}$; il en va de même en France à la même époque selon Charles Tilly. Tout cela suggère de prendre au sérieux ce que l'on appellera ici, faute de mieux, la "culture manifestante" des différents groupes sociaux, culture qu'il faut mettre évidemment en rapport avec des répertoires d'action stéréotypés et différenciés. A observer notre corpus, il semble bien que certains groupes sociaux aient des répertoires d'action spécifiques. La construction de barricades, pour s'en tenir à un exemple, n'est actuellement utilisée que par les étudiants, à quelques rares exceptions près, à la fois sans doute en référence aux journées révolutionnaires traditionnelles et à 1968 mais aussi parce que les étudiants se regroupent souvent dans les bâtiments de leurs 
universités, soit pour les occuper, soit pour s'y réfugier. Le sentiment de disposer d'un "territoire" devant rester libre de toute intervention policière explique alors le blocage des rues. C'est ainsi qu'en 1986, à la fin de la manifestation du 4 décembre aux Invalides, les derniers manifestants se regroupent et se rendent naturellement dans le quartier latin pour se réfugier et faire le point. Puis, le 6 décembre, à l'issue de la manifestation en hommage à Malik Oussekine, c'est dans le quartier Saint Michel que plusieurs barricades sont dressées et les manifestants tiennent la rue de $22 \mathrm{~h} 30$ à 2 heures du matin ${ }^{31}$. Cette spécificité des manifestations étudiantes n'est pas proprement parisienne comme en témoigne une manifestation "exemplaire" des étudiants en médecine, le 19 mai 1983 à Montpellier. Mobilisés contre le projet de réforme des études médicales, 500 à 600 étudiants dressent tôt le matin autour du bâtiment de leur faculté, située en pleine ville, trois grosses barricades destinées à empêcher l'accès aux bâtiments et à bloquer la circulation sur un carrefour. Les barricades se composent d'épaves de véhicules accidentés, de barrières, de fils de fer barbelés, de pneus, le tout étant copieusement arrosé d'essence pour être enflammé au cas ou la police interviendrait. Derrière les barricades les manifestants s'organisent militairement et stockent des munitions pour résister à l'assaut. Galets, bouteilles d'essences, ampoules pleines de peinture et cocktails molotov sont préparés. "Une rue est dépavée comme au temps de 1968", relate la presse. A partir de 17 heures, un haut parleur avertit les manifestants que les forces de l'ordre vont intervenir. Les étudiants reçoivent l'assurance préalable que la faculté serait considérée comme zone neutre à condition que l'on ne lance pas de projectiles des toits. Après un bref assaut, les étudiants se réfugient dans les bâtiments et tout rentre dans l'ordre. On dénombre quelques blessés du côté des manifestants et des forces de l'ordre. Cet exemple illustre bien un certain type de manifestation propre aux étudiants, revendiqué d'ailleurs comme tel par les étudiants eux-mêmes, analysé par la presse comme troubles typiquement étudiants (avec, dans chaque compte-rendu, le rappel de 1968) et géré de façon spécifique par les forces de l'ordre ${ }^{32}$. Tout concourt ici à entretenir et à marquer une tradition manifestante caractéristique, à tel point que l'on peut se demander si dans ce cas il ne faudrait pas parler de ritualisation des comportements. Dans notre exemple, les étudiants n'ont pas l'intention d'avoir recours aux éléments de défense mis en place (comme par exemple l'incendie des barricades) ${ }^{33}$. Mais la réalisation d'un dispositif sophistiqué contribue à la dramatisation de la situation, à entretenir "l'identité étudiante", et joue un rôle non négligeable auprès des média qui sont complaisamment invités à "visiter" les lignes de défense. Enfin, le déclenchement de l'action policière est précédé d'une négociation sur les limites du jeu, offrant aux étudiants le plaisir d'une résistance et aux forces de l'ordre l'assurance d'un retour à l'ordre sans véritable affrontement. On voit encore dans cet exemple combien la forme prise par la violence dépend étroitement des relations qui s'instaurent à l'avance ou dans l'action entre les protagonistes. Aussi apparait-il nécessaire de faire porter également l'analyse sur l'autorité civile et les forces de l'ordre. Nous nous en tiendrons ici à montrer l'importance de la perception du groupe protestataire par les autorités et l'existence de modes de gestion différenciés des conflits.

Les facteurs liés à l'autorité civile et aux forces de l'ordre Nous avons souligné plus haut l'importance des processus de communication dans l'interaction entre les agents et dans la probabilité événements violents. Cette communication peut s'analyser comme un "jeu d'assurance", c'est-à-dire comme un échange dans lequel l'action dépend de l'anticipation des coups de l'adversaire ${ }^{34}$ : les acteurs modulent leurs actes 
en fonction de ce qu'ils croient pouvoir être toléré par les forces de l'ordre en même temps que l'autorité civile fixe, implicitement ou explicitement, des seuils de tolérance en fonction de la nature des protagonistes. La perception des groupes de manifestants par les autorités n'est à l'évidence pas homogène, qu'il s'agisse des autorités politiques ou des forces de l'ordre sur le terrain. Il est donc pertinent de chercher à mettre en rapport le niveau de violence dans la manifestation avec le degré de répression policière. Toutefois une investigation de ce type pose un certain nombre de problèmes que nos sources ne sauraient résoudre. Aussi s'en tiendra-t-on ici à une approche moins systématique que nous ne l'aurions souhaitée, en insistant sur certains aspects révélés par le dépouillement des rapports techniques.

Perception du groupe par les autorités et les forces de l'ordre : le cas le moins douteux où les forces de l'ordre, sur ordre de l'autorité civile, ont une attitude nettement répressive vis-à-vis des manifestants est celui des manifestations interdites non tolérées. La manifestation des opposants au régime turc à la Concorde, lors de la visite de Tugurt Ozal à Paris le 30/11/88, illustre bien ce point. Les organisations ayant appelé à manifester prévoyaient un rassemblement devant l'hôtel Crillon, place de la Concorde, pour conspuer Tugurt Ozal, invité officiellement par la France. Les instructions de l'autorité civile sont fermes: les forces de l'ordre ont pour consigne d'empêcher tout rassemblement sur la place et de procéder à des arrestations. Dès dix heures, les manifestants commencent à sortir par petits paquets du métro. Bientôt une trentaine de personnes se rassemblent près de l'obélisque. Le commandant de la CRS reçoit alors l'ordre de procéder à des arrestations, ce qu'il refuse de faire faute de moyens (absence de cars). Une demi-heure plus tard les opposants sont environ deux cents et devant le mouvement des forces de l'ordre pour les disperser, les premiers heurts commencent (les manifestants sont porteurs d'armes par destination). Une cinquantaine d'interpellations sont effectuées. A dix heures vingt cinq, les sommations sont effectuées pour disperser le reste des manifestants qui s'accroupissent alors. L'intervention des forces de l'ordre bloque les manifestants contre les grilles de l'obélisque ce qui permet de procéder à un grand nombre d'interpellations. Pendant cette opération, des conteneurs lacrymogènes sont utilisés pour neutraliser les plus virulents. Puis une manoeuvre de dégagement à l'initiative des forces CRS permet aux manifestants de se disperser vers le quai des Tuileries. A 10 heures quarante cinq tout est terminé. 14 blessés sont recensés parmi les forces de l'ordre, un manifestant, "indisposé par le gaz lacrymogène s'est affaissé et a été conduit par les civils de la préfecture de police au pied des grilles de l'Obélisque, grilles auxquelles ils l'ont menotté par un poignet, jusqu'à l'arrivée d'un fourgon de premier soins" signale le rapport CRS. Les interpellations sont très nombreuses puisque plusieurs cars de la préfecture de police sont mobilisés. On a là un bon exemple des différences de traitement dont les manifestants font l'objet en fonction des instructions données par l'autorité civile: l'intervention des forces de l'ordre a précédé toute violence manifestante, étant donné les consignes de dispersion immédiate ; contrairement à la plupart des opérations de maintien de l'ordre, le but des manoeuvres étaient moins de procéder à la dispersion des manifestants que de procéder à des arrestations. D'où le blocage des opposants contre les grilles de l'obélisque, procédé sur lequel le rapport technique émet d'ailleurs quelques réserves étant donné les violences qui en ont résulté; les sommations n'ont été effectuées qu'après une première vague de refoulement et une cinquantaine d'arrestations ; enfin, des fonctionnaires en civil de la 
préfecture de police sont sur place pour "filtrer la presse", afin d'éviter une trop grande publicité.

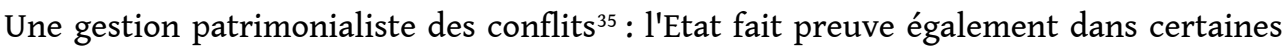
circonstances d'une grande tolérance face à l'illégalisme des manifestants. Sur l'ensemble des manifestations violentes recensées dans les rapports techniques $(\mathrm{N}=$ 152) , 28 font l'objet d'une "gestion douce" par les forces de l'ordre, sur instruction de l'autorité civile. Dans la plupart des cas, il s'agit de manifestations agricoles puis, dans une moindre mesure, étudiantes. D'abord, il n'est pas rare que lors des négociations entre les groupes organisateurs et les autorités avant une manifestation soient définies des "zones de tolérance" dont la fonction est de délimiter à l'avance le type et le degré de violence qui ne donneront pas lieu à une intervention des forces de l'ordre. Cette délimitation de degrés d'acceptabilité dans l'illégalisme remplit une double fonction. Elle permet d'abord à l'autorité civile de définir les cibles acceptables de la violence et d'en écarter d'autres jugées plus sensibles ou plus coûteuses, comme par exemple des bâtiments publics, des biens privés, etc. ${ }^{36}$. Elle permet en même temps aux organisateurs de ne pas se couper d'une base quelquefois décidée à en découdre coûte que coûte et donc d'en garder le contrôle. La gestion patrimonialiste des conflits permet encore à l'autorité civile, dans une perspective stratégique, de déterminer à l'avance les cibles visées par les manifestants, notamment pour éviter la destruction de biens privés: lors de la journée "agriculture morte" organisée dans les PyrénéesOrientales le 25 janvier 1982 par la FNSEA et le CDJA pour protester contre les importations en provenance d'Espagne, les manifestants ont l'intention d'interdire toute circulation ainsi que toute vente de produits agricoles. L'objectif principal de la journée est d'intercepter tous les camions étrangers et d'en déverser le contenu sur la chaussée. Aussi, lors de la réunion préparatoire à la préfecture, un certain nombre de mesures préventives sont-elles prises: en accord avec les autorités espagnoles, la frontière est fermée à plusieurs endroits et les camions espagnols sont retenus aux villes frontières. De plus, aucun train de marchandise ne doit circuler ce jour là. Prévoyant la colère des agriculteurs devant l'absence de camions à arraisonner, les forces de l'ordre ont pour consigne "d'observer" les dégradations qui seront commises et de n'intervenir qu'en cas d'urgence. Le 25, dès 9 heures, des barrages filtrants sont mis en place sur les autoroutes par les agriculteurs. La situation reste calme jusqu'à 12 heures. A partir de ce moment, les manifestants comprennent que des mesures de prévention ont été prises et qu'ils n'intercepteront pas de camions espagnols. Aussi, un camion transportant du gravier émulsionné est-il arrêté et son chargement déversé sur la voie ferrée. Une demi-heure plus tard, c'est un chargement de béton liquide qui est renversé sur la même voie. Les forces de l'ordre restent en retrait et n'interviennent pas. Un hélicoptère de la gendarmerie est chargé de la surveillance. De 13 heures à 14 heures, 600 mètres de rails sont arrachés à l'aide de tracteurs, ce qui provoque une intervention des CRS. Les manifestants se dispersent immédiatement et le préfet donne instruction de ne pas poursuivre les manifestants et de ne procéder à aucune interpellation. Un peu plus tard, des arbres sont coupés à la tronçonneuse et la chaussée est arrachée sur cinq mètres à Saint Genis des Fontaines. Enfin, vers 16 heures, les manifestants se regroupent devant la préfecture et exigent de pouvoir défiler devant le bâtiment et d'y déposer un tronçon de voie ferrée. Le préfet demande alors à recevoir une délégation, et, sur l'engagement solennel du responsable qu'il n'y aura pas de dégradation, le préfet autorise les manifestants à défiler. Le barrage est ouvert et une partie du personnel CRS embarque dans les cars, la présence des forces de 
l'ordre étant perçue comme une "provocation". Le tronçon de voie est projeté contre la guérite de verre du gardien qui se brise puis la dislocation commence. A la fin de l'opération, une voiture des polices urbaines avec cinq personnes à bord est renversée par les derniers manifestants. A l'issue de la manifestation, le préfet réunit les différents chefs de service concernés et se félicite du bon déroulement de la manifestation, "en ne cachant pas que pour lui les 1.200 .000 francs de dégâts au préjudice de la SNCF étaient un moindre mal, car des incendies de camions, à 500.000 francs par camion, auraient pu coûter infiniment plus cher". Ce récit est révélateur de la tolérance dont peut faire preuve l'autorité civile vis-à-vis de manifestants très violents et prêts à se livrer à d'importantes déprédations. Malgré le déversement de plusieurs chargements d'origine non agricole, l'endommagement de routes et la destruction d'une partie de la voie ferrée, le préfet demande à recevoir une délégation, l'autorise à défiler devant la préfecture et accepte même de retirer les forces de l'ordre. Cette attitude n'est bien entendu pas le fruit d'une sympathie marquée de l'autorité civile pour les agriculteurs mais bien plutôt le résultat d'un calcul d'utilité destiné à minimiser le plus possible les déprédations. Et ce calcul se fait plus ou moins en concertation avec les organisateurs, toujours inquiets des possibilités de débordements de la base. Dans le cas de cette manifestation, les organisateurs avaient prévenu l'autorité de la présence dans leurs rangs d'éléments "jeunes" difficilement maitrisables... Ce dernier point indique bien que "l'équilibre" recherché au cours du déroulement d'un conflit est extrêmement fragile et que tout peut basculer rapidement sous l'effet d'un geste malheureux, d'une intervention "prématurée".

11 Analyse de quelques facteurs morphologiques Si la violence est contrainte, et partiellement déterminée, par les caractéristiques des groupes, il faut encore s'arrêter aux propriétés des événements eux-mêmes à travers leurs caractères morphologiques. Nous n'en analyserons que quelques uns ici, étant donné la grande diversité des processus à envisager.

12 Le nombre et les répertoires d'action: on considère généralement que toute manifestation qui rassemble un nombre élevé de participants n'est plus entièrement contrôlable, le seuil se situant assez bas, aux alentours de quelques milliers de personnes. Il y aurait deux raisons principales à cela. D'une part, une raison purement morphologique : la masse des manifestants représente une force physique qui peut être considérable et qui dispose parfois d'un tel avantage numérique sur les forces de l'ordre qu'elle n'est guère canalisable. Par ailleurs, le nombre accroît la visibilité de la manifestation et donc la probabilité que s'introduisent dans le cortège des éléments enclins à l'action violente. D'une certaine façon, on n'est pas loin ici de la théorie de l'impunité développée par les tenants de la psychologie des foules selon laquelle plus le nombre de participants à une action est élevé, plus le sentiment que l'on ne risque rien pousse à la violence et aux exactions diverses. Or, dans notre corpus, les manifestations importantes sont sur-représentées dans les manifestations violentes par rapport aux manifestations recensées dans les télégrammes en 1989 comme en 1990 : 13\% des 152 manifestations violentes ont regroupé plus de 5000 personnes contre seulement $4 \%$ des télégrammes en 1989 et 7\% en 1990. La même chose est vraie, dans une moindre mesure cependant, pour les manifestations comprises entre 1001 et 5000 personnes. A l'inverse, les petites mobilisations de moins de 200 personnes ne sont que $17 \%$ dans notre échantillon de manifestations violentes, contre respectivement $46 \%$ et $43 \%$ en 1989 et 1990. Pour préciser cet effet du nombre, il faut encore tenir compte du répertoire d'action, ce qui permet de préciser si la corrélation entre accroissement du nombre et 
fréquence des violences détermine une causalité indépendante ou dépendante des modes d'action. On observe alors que les manifestations violentes s'inscrivent généralement dans des répertoires d'action assez caractéristiques: les défilésrassemblements violents rassemblent beaucoup plus de monde que les non-violents. Il y a donc bien là un effet de taille; les rassemblements statiques sont rarement l'occasion de violences, quelque soit le nombre impliqué ; les obstructions, opérations commandos et occupations se traduisent plus souvent que d'autres modes d'action par des violences, et ce d'autant plus que le nombre de participants est élevé. Par ailleurs, à chaque mode d'action est associée une forme et une intensité particulière de violence. Si les défilés de masse se traduisent la plupart du temps par des affrontements entre forces de l'ordre et manifestants empêchés de progresser, les occupations de locaux sont plutôt marquées par des opérations d'évacuation opposant manifestants retranchés et forces de l'ordre, etc. Les répertoires d'action utilisés sont donc bien déterminants de la survenance des violences, de leur forme et de leur intensité.

13 L'itinéraire : Le fait que le point d'aboutissement de la manifestation soit le lieu où s'exerce l'autorité qui a le pouvoir de décision ou un lieu symbolique a généralement plus de chance de provoquer des incidents que s'il s'agit d'un lieu de dispersion neutre. Il semblerait que les manifestants aient toujours plus de mal à se disperser lorsqu'ils ont la possibilité (ou l'illusion de la possibilité) de faire pression directement sur le lieu de pouvoir compétent ou mis en cause. Toutefois, étant donné qu'en province nos manifestations se déroulent ou aboutissent dans la majorité des cas devant une préfecture ou une sous préfecture, il est difficile d'isoler là un facteur indépendant de violence. En revanche, les modifications d'un itinéraire en cours de manifestation ont toutes les chances de provoquer des affrontements. La manifestation lycéenne du 12 novembre 1990, bloquée au dernier moment au pont de l'Alma, en est une bonne illustration. Dans ce cas, la décision de stopper le cortège avant le lieu de dispersion prévu, l'impossibilité dans laquelle se trouvaient les responsables, des organisations lycéennes mais également des forces de l'ordre, d'expliquer les raisons de cette décision, sont à l'origine des violences, violences, soulignons le, qui au départ ne sont pas le fait de "casseurs" ou "d'autonomes". On peut également relever un certain nombre de situations dans lesquelles l'interdiction faite à un défilé de "rééditer" une action précédente provoque l'incompréhension des manifestants et, par là même, des violences. Lors d'une manifestation des salariés des aciéries du Tarn à Albi le 19 juillet 1982, les manifestants investissent pacifiquement la préfecture et l'occupent toute la matinée. Aucun heurt n'est à signaler. Le lendemain, les sidérurgistes reviennent tôt le matin avec l'intention de réoccuper la préfecture. Mais des dispositions ont été prises et les grilles fermées sont protégées par un cordon de CRS. Le rédacteur du rapport technique écrit:"Les manifestants avaient pour idée première de réitérer leur opération commando de la veille et ont, au départ, trouvé drôle que les pouvoirs publics s'opposent à leur action "puisque tout s'était bien passé la matinée précédente". Certains d'entre eux ont voulu engager la polémique avec le personnel de l'unité se trouvant dans le sas de l'entrée principale mais se sont heurtés au mutisme de ce dernier. Ils ont alors couvert d'injures les fonctionnaires de police (...) Par la suite, les manifestants, qui venaient de se rendre compte que leurs tentatives de pénétration dans l'édifice étaient vouées à l'échec, ont mitraillé les CRS à l'aide de boules puantes puis d'oeufs, de tomates et de petits cailloux. Dans le même temps quelques uns essayaient de blesser des fonctionnaires en se servant de grands bouts de bois comme piques". 
La répétition des manifestations : la répétition des manifestations est la plupart du temps corrélée avec l'apparition de violences. Que l'on songe par exemple aux manifestations étudiantes de 1983 contre la loi Savary, au conflit des fonctionnaires corses qui dure du 23 mars au 18 avril 1989 et culmine avec la manifestation particulièrement violente du 12 avril, etc. Dans les conflits longs, la violence apparaitt comme le fruit de logiques différentes: d'abord, pour éviter la lassitude du public, les organisations ont intérêt à "occuper le terrain médiatique" en sacrifiant à une logique du spectaculaire. Il peut s'agir aussi d'une logique d'escalade, la violence devenant un moyen de marchandage avec les pouvoirs publics. Enfin, plus simplement, on peut évoquer un effet de "ras le bol" devant une mobilisation longue qui tourne court sans avoir débouché sur des concessions satisfaisantes. Nous n'en donnerons qu'un exemple, révélateur en ce qu'il touche une mobilisation particulièrement peu violente: le mouvement des agents des finances en $1989^{37}$. Le mouvement dure de juin à décembre 1989, ponctué par un grand nombre de manifestations à Paris comme en Province. On peut distinguer trois phases dans la mobilisation avec d'abord, de juin à août une série d'actions limitées, puis de septembre à octobre, une mobilisation de masse qui concerne la plupart des agents des finances, précédant en novembre-décembre un essoufflement du mouvement. Le graphique infra illustre ces trois phases par la répartition mensuelle du réservoir de mobilisation de juin à décembre (les données proviennent du dépouillement des télégrammes de 1989).

\section{Nombre de personnes descendues dans la rue durant le conflit}

Or, c'est justement pendant cette période de perte de vitesse du mouvement, alors même que les syndicats n'ont pratiquement rien obtenu de leurs revendications, que les violences font leur apparition. Le 15 novembre, un rassemblement statique des fonctionnaires des impôts à proximité de l'Assemblée nationale se termine par un défilé non prévu à l'avance. Le cortège se rend rue de Solférino au siège du PS, la porte du bâtiment est enfoncée et deux permanents du parti sont blessés. Au même moment, un groupe essaie de se rendre à l'Elysée et, arrêté par les CRS au début de l'avenue de Marigny, des coups sont échangés. On est bien là en présence d'un cas où la lassitude des manifestants alliée à la certitude d'avoir "perdu" est à l'origine des débordements.

L'hétérogénéité des forces en présence et les rivalités internes: les deux tiers des manifestations de notre corpus (106) sont organisées à l'appel de trois ou plus de trois organisations. C'est que lorsque plusieurs groupes sont impliqués, les stratégies, et notamment la stratégie de recours à la violence, peuvent varier, les mots d'ordre peuvent être contradictoires. Lors de la fête annuelle basque Aberri Eguna-jour de la patrie, d'avril 1990, par exemple, une manifestation à lieu à Hendaye, marquée par un certain nombre d'actions symboliques à caractère pacifique. Après le rassemblement pendant lequel est lue publiquement une lettre à P. Joxe, suivie de plusieurs prises de parole, un cortège de 2000 personnes (dont beaucoup de femmes et d'enfants) s'avance sur le pont Saint-Jacques et jette solennellement une borne frontière en carton dans la Bidassoa. A ce moment, la tête du cortège est débordée par environ 300 manifestants du groupe nationaliste Patcha Agir qui se placent entre les forces de l'ordre et le défilé pour jeter des pierres sur les CRS. Des drapeaux français et espagnols sont brûlés, ce qui provoque une charge destinée à récupérer les emblèmes nationaux, puis une dispersion 
musclée après que le gros des manifestants se soient retirés. Cinq blessés sont recensés. Dans ce cas, les différences de sensibilité et de stratégie des manifestants sont à l'origine des violences. Du côté des forces de l'ordre, enfin, l'hétérogénéité des corps de police engagés dans une opération de maintien de l'ordre peut être à l'origine de violences, du fait d'une mauvaise coordination ou de méthodes divergentes. Par exemple, lors de la manifestation étudiante du 19 mai 1983 à Montpellier dont nous avons déjà examiné les circonstances plus haut, l'intervention simultanée des CRS, des gendarmes et des policiers urbains a eu pour effet de provoquer une grande confusion dans les rangs des CRS, et des charges un peu erratiques (chaque équipe ayant tiré des lacrymogènes dans des directions différentes, le terrain était difficilement praticable). Le rapport technique déplore que chaque force de maintien de l'ordre engagée ait suivi sa propre stratégie et ait utilisé des moyens de nature différente. En effet, si les CRS et les polices urbaines utilisent des grenades détonatrices et lacrymogènes lors de la charge, les gendarmes ont utilisé quelques grenades offensives rendues responsables de plusieurs blessés par la presse et le rapport. Ce type de situation confuse reste toutefois l'exception, mais il est fréquent que les commandants de compagnies se plaignent des effets néfastes de l'interpénétration des forces.

\section{CONCLUSION}

Nous espérons avoir montré, à travers cette brève présentation des dossiers CRS, que la violence s'inscrit dans un continuum de possibilités tactiques, constituant un recours normal de l'action collective, bien que son utilisation ne réponde pas toujours aux mêmes visées (élément de marchandage politique dans l'échange de coups ou instrumentalisation à des fins identitaires dans d'autres). Cela ne signifie pas pour autant que la survenance des violences ressort toujours de plans déterminés à l'avance, les protagonistes n'en maîtrisant jamais tout à fait l'apparition et/ou les effets dans les processus d'interaction. L'engagement dans la violence est discontinu dans le temps et dépend lourdement de facteurs situationnels, ce qui plaide pour la nécessité de faire porter d'abord l'analyse sur les processus. Parmi ces facteurs, nous avons identifié la nature des interactions entre les adversaires et le poids des contextes (facteurs morphologiques). Le premier implique que l'on mette sur le même plan la stratégie des différents protagonistes (manifestants, contre-manifestants et forces de l'ordre), au nom d'une identité de nature. Le second nous rappelle que les mobilisations sont d'abord une forme d'action et non pas un ensemble de "dispositions à". L'analyse de l'émergence de la violence peut donc s'enrichir de l'étude de la structure des opportunités du moment, sachant que le spectre entier des stratégies disponibles dans un environnement politique dynamique dépend à la fois de l'environnement externe et de l'environnement interne des groupes. Le premier détermine le potentiel stratégique disponible pour chaque groupe en fonction de variables comme le statut du groupe dans son environnement, le niveau de coercition admissible, les contraintes politiques pesant sur les responsables de l'ordre, etc. L'étude de l'environnement interne, quant à elle, permet d'expliquer pourquoi, dans le champ des possibles stratégiques, tel mouvement choisit tel mode d'action plutôt que tel autre. Il faut alors insister sur la structure organisationnelle des mouvements, sur la nature du leadership, sur la perception de leur environnement par les membres du groupe mobilisé, sur le poids 
des traditions et sur l'expérience antérieure, lesquels déterminent en partie les répertoires d'action utilisables. Nous en avons rapidement esquissé ici quelques traits à travers l'analyse de l'influence des "cultures manifestantes" sur les répertoires d'action. Enfin, il est important de rejeter définitivement le postulat d'une hétérogénéité des causes (déterminants en amont, états initiaux) et de leurs produits (les événements violents, les résultats). C'est en termes de processus que l'on doit penser les enchaînements causaux, processus par lesquels les actes et les structures de l'action se mettent en place et se modifient au fur et à mesure de l'échange de coups.

\section{NOTES}

1. Nous pensons d'abord ici aux deux modèles dominants en sociologie de la mobilisation, à savoir celui de la privation relative (Gurr, Why Men Rebel, Princeton, Princeton University Press, 1970) et celui de la mobilisation des ressources, Oberschall (A.), Social Conflict and Social Movements, Prentice Hall, Englewood Cliffs, New Jersey, 1973 ; Charles Tilly, From Mobilization to Revolution, Addison Wesley, 1978, mais la critique vaut tout autant pour le modèle structuro-fonctionnaliste de Smelser in Theory of Collective Behavior, New York, Free Press, 1962, et les travaux marxistes et néo-marxistes.

2. Gurr, Why Men Rebel, op. cit. ; Snyder (D.), Tilly. (C.), "Hardship and Collective Violence in France : 1830-1960", American Sociological Review 37, 1972, pp 520-532 ; Gurr, "A Comparative Study of Civil Strife", in Graham \& Gurr. (eds) : Violence in America, Historical and Comparative Perspectives, Washington D. C, US Government Printing Office, 1969 ; Hibbs, Mass Political Violence : A Cross-National Causal Analysis, NY, Wiley, Interscience, 1973.

3. Mass Political Violence : A Cross-National Causal Analysis, op. cit.

4. Gurr (T. R.), "A Comparative Study of Civil Strife", in Graham \& Gurr (eds) : Violence in America, Historical and Comparative Perspectives, Washington D. C, US Government Printing Office, 1969.

5. Tilly (C.), "Revolutions and Collective Violence", in Greenstein \& Polsby (eds) : Handbook of Political Science, Vol 3, Reading Mass, Addison Wesley, 1975.

6. Davies (J. C), "Toward a Theory of Revolution", American Sociological Review, $n^{\circ} 23$, 1962, pp 5-19.

7. Hibbs, op. cit.

8. La recherche porte ici sur deux types de documents d'archives du ministère de l'Intérieur : les télégrammes d'emploi dressés à l'occasion de chaque emploi des CRS et les rapports circonstanciés (ci-après Rapports Techniques) rédigés par les commandants de compagnie lorsque une opération de maintien de l'ordre sort du cadre routinier. Ces rapports concernent principalement la province, étant donné les logiques d'emploi des compagnies. Nous avons eu accès à ces sources dans le cadre d'un contrat de recherche avec l'IHESI, mené en commun avec Pierre Favre ; Cf. Pierre Favre et Olivier Fillieule, Manifestations pacifiques et manifestations violentes dans la France contemporaine, (1982-1990), rapport à l'IHESI, 1992. 
9. Par le choix de sources documentaires nous interdisant ce type d'inférences causales, nous espérons pouvoir nous garder de ce que Michel Dobry a nommé l'illusion étiologique, Sociologie des crises politiques, Paris, Presses de la FNSP, 1986, p 50. 10. Cf. Schelling, The Strategy of Conflict, Cambridge, Oxford. 1960 ; Stratégie du conflit, Trad., PUF, 1986.

11. Contrairement à ce que suggère Goffman (1974), nous ne pensons pas que l'échange de coups dans une mobilisation se ramène à une interaction stratégique pure dans laquelle il n'y aurait pas d'échange d'information, de communication entre les protagonistes de l'interaction. Tout d'abord, si les acteurs règlent leur conduite à partir d'une estimation du jeu de l'autre (c'est-à-dire l'aspect expressif de son comportement), il produisent également des symboles et des codes à l'intention de l'adversaire, ce qui constitue une forme de communication. Mais surtout, il faut tenir compte, tout au moins dans les manifestations de notre corpus, de la place considérable des rapports de "face à face" et des procédures de coopération .

12. Michaud (Y.), Violence et politique, Paris, Gallimard, 1978.

13. Cf. sur ce point la critique que fait Walsh "On the Interaction Between a Movement and its Environment (Comment on Jenkins and Perrow)", American Sociological Review, vol 43, N 1 , février Juin, 1978. de J. C. Jenkins et C. Perrow, "Insurgency of the Powerless : Farm Worker Movement (1946-1972)", American Sociological Review, n42, pp 249-268, 1977).

14. Oberschall (A.), Social Conflict and Social Movements, op. cit.

15. Cf. M. Dobry, op. cit., p 25-26.

16. Cf. D. Monjardet, "La Manifestation du côté du maintien de l'ordre", in P. Favre, La Manifestation, Paris, Presses de la FNSP, pp 207-228, 1990.

17. On en a une bonne illustration dans la classique distinction entre la violence et la force, que l'on trouve aussi bien chez Hobbes, Locke ou Hegel. Pour ces auteurs, la force est considérée comme éthiquement neutre et la violence comme irrationnelle et excessive. Au fond, on débouche sur l'opposition webérienne entre la violence légale et légitime et la violence illégale et illégitime, l'Etat détenant le monopole de la première. 18. L'inclusion des opérations de qualification de sa propre action et de celle de l'adversaire dans l'échange de coups nous est suggérée par M. Dobry, op. cit., 1986, p 33. 19. Zimmerman (E.) Political Violence, Crises and Revolutions. Theories and Research, Cambridge, Schenkman, 1983.

20. C'est nous qui soulignons. Nieburg (H. L.), Political Violence, The Behavioral Press, New York, St'Martin's Press, 1969, p 13), cité in Zimmerman (E.) Political Violence, Crises and Revolutions. Theories and Research, op. cit., 1983, p 8.

21. Rappelons que les télégrammes rendent compte de l'ensemble des interventions CRS sur le territoire.

22. Ce qui confirme l'hypothèse émise par Hibbs, op. cit., selon laquelle une exclusion effective des positions dominantes en termes ethniques, religieux ou linguistiques se traduit généralemment par des formes adoucies de protestation. Cf. aussi Gurr, "A Comparative Study of Civil Strife", op. cit., p 71, pour la même remarque.

23. Franck (J.A) ,"La dynamique des manifestations violentes", Revue canadienne de science politique, 17 (2), juin, 1984, pp 325 - 349.

24. Ce qui n'empêche qu'il y ait toujours instrumentalisation de la violence, mais moins dans le but de négocier que dans celui d'affirmer l'identité du groupe.

25. Wilson (J. Q.), Political Organizations, New York, Basic Books, 1973. 
26. Cf. les travaux de Snow sur les "frame alignment" et de Klandermans sur la mobilisation du consensus : Snow (D. A.) et Benford (R.) ," Ideology, Frame Resonance and Participant Mobilization", in Klandermans, Kriesi, Tarrow. (eds) : From Structure to Action : Comparing Social Movements Across Cultures, Greenwich, Conn, JAI, 1988, pp. 197-218 ; Klandermans (B.) , "The Formation and Mobilization of Consensus", ibidem, pp 173-196.

27. De Montalibert (C.), Crise économique et conflit sociaux dans la Lorraine sidérurgique, Logiques sociales, L'Harmattan, 1989.

28. Les forces de l'ordre et les représentants de l'autorité civile sont pénétrés de cette idée selon laquelle les nouveaux groupes de manifestants constituent un danger supplémentaire. C'est le cas notamment des manifestations de jeunes et, plus particulièrement, de lycéens dont Maurice Grimaud (En Mai fais ce qu'il te plait, Paris, Stock, 1977) avait constaté avec inquiétude l'apparition dans les manifestations le 10 mai 1968 et souligné que "si nous devions les retrouver chaque fois devant nous combien plus délicates encore allaient devenir nos interventions" (p 162). Aussi bien, la commission d'enquête sénatoriale sur les manifestations de décembre 1986 insistaitelle également sur les risques dûs à la présence dans le mouvement étudiant d'une forte proportion de lycéens inexpérimentés (plus des deux tiers). Cf. le rapport de Paul Masson Rapport fait au nom de la commission d'enquête créée en vertu d'une résolution adoptée par le Sénat le 17 décembre 1986, chargée de recueillir des éléments d'information sur la préparation, l'organisation, le déroulement et la présentation des évènements de novembre et décembre 1986, 270, Sénat, juin 1987.

29. Voir par exemple G. Rudé, The Crowd in History, 1730-1848, New York, Wiley, 1964, chapitre 6 et 11 ; Tilly (C.),"Reflexions on the Revolutions of Paris : An Essay on Recent Historical Writings", Social Problems, XII, 1964, pp 99-212.

30. In Rudé, op. cit., chapitre 2 ; Hobsbawm, Social Bandits and Primitive Rebels, New York, The Free Press, 1959, chapitres 4 et 5, décrit de la même façon le développement d'une tradition de violence millénariste au sein de la paysannerie du sud de l'Europe comme réponse stéréotypée aux privations endémiques.

31. Cf. aussi le 24 mai 1983, l'importante manifestation des étudiants contre le projet de loi Savary.

32. Cf. par exemple l'accord préalable sur la désignation de la faculté comme zone neutre.

33. Ce qui n'empêche évidemment pas que puisse survenir un élément aléatoire ou inconnu des manifestants dont l'effet serait le recours effectif aux éléments de défense et d'attaque préparés (que l'on songe par exemple à la présence d'un individu ou de groupes particulièrement excités, à l'action d'un agent provocateur).

34. Le concept de jeu d'assurance, illustré notamment par Elster (J.) (Ulysses and the Sirens, Cambridge, Cambridge University Press, 1969) s'applique traditionnellement aux relations entre acteurs d'un même groupe potentiel pour expliquer les motivations de la participation.

35. Nous empruntons l'expression à Patrice Mann, L'Activité tactique des manifestants et des forces mobiles lors des crises viticoles du midi (1950-1990). Contribution à l'étude de la dynamique des répertoires d'action collective, rapport dactylographié, IHESI, 1991.

36. Ce type de négociation s'explique par la responsabilité financière de l'Etat en matière d'atteinte aux biens publics ou privés dans le cadre des manifestations. L'article 92 de la loi du 7 janvier 1983 stipule en effet que "l'Etat est légalement 
responsable des dégâts et dommages résultant des crimes et délits commis, à force ouverte ou par violence, par des attroupements ou rassemblements armés ou non armés, soit sur les personnes soit sur les biens". Cette responsabilité ouvre aux préfets la possibilité de choisir, selon divers critères, le type de violence acceptable à la fois en termes symboliques et financiers

37. Sur ce mouvement, on consultera avec profit le travail de Polac (C.), Protestation et crédibilité des agents des finances. Etude du mouvement de mai à novembre 1989, mémoire pour le DEA de sociologie politique,.sous la direction de Philippe Braud, Université Paris I, 1992.

\section{RÉSUMÉS}

L'engagement dans la violence est discontinu dans le temps et dépendant lourdement de facteurs situationnels, ce qui plaide pour la nécessité de faire porter l'analyse sur les processus. Nous avons en effet choisi de limiter l'analyse au moment même de la manifestation, au nom d'une orientation théorique centrée sur l'échange de coups plutôt que sur les prédéterminations. Dans cette perspective, nous montrons, à travers l'analyse de dossiers de police, que la violence est le plus souvent le produit d'un produit d'un processus itératif d'adaptation tactique de chacun des protagonistes, ce qui implique que l'on mette sur le même plan les stratégies des mouvements et celles de l'Etat (autorité civile et policière) au nom d'une identité de nature. Un certain nombre de facteurs déterminants des violences sont identifiés tant du côté des manifestants (nature des objectifs et répertoires d'action) que des autorités (perceptions du groupe par les forces de l'ordre, gestion patrimonialiste). Enfin, l'analyse suggère qu'il est également nécessaire de prendre en compte un certain nombre de facteurs morphologiques tels que le nombre de manifestants, la succession des actions dans le temps et l'hétérogénéité des forces de présence.

\section{INDEX}

Mots-clés : Mouvements sociaux, sciences politiques, violence, émeutes 